\title{
LOCAL ENERGY ESTIMATES FOR THE FINITE ELEMENT METHOD ON SHARPLY VARYING GRIDS
}

\author{
ALAN DEMLOW, JOHNNY GUZMÁN, AND ALFRED H. SCHATZ
}

\begin{abstract}
Local energy error estimates for the finite element method for elliptic problems were originally proved in 1974 by Nitsche and Schatz. These estimates show that the local energy error may be bounded by a local approximation term, plus a global "pollution" term that measures the influence of solution quality from outside the domain of interest and is heuristically of higher order. However, the original analysis of Nitsche and Schatz is restricted to quasi-uniform grids. We present local a priori energy estimates that are valid on shape regular grids, an assumption which allows for highly graded meshes and which matches much more closely the typical practical situation. Our chief technical innovation is an improved superapproximation result.
\end{abstract}

\section{INTRODUCTION}

In this paper we prove local energy error estimates for the finite element method for second-order linear elliptic problems on highly refined triangulations. Most a priori error analyses for the finite element method in norms other than the global energy norm place severe restrictions on the mesh. In particular, such error analyses are most often carried out under the assumption that the grid is quasi-uniform, that is, all simplices in the mesh are required to have diameter equivalent to some fixed parameter $h$. The typical practical situation is rather different. Many (especially adaptive) finite element codes enforce only shape regularity of elements, meaning that all elements in the mesh must have bounded aspect ratio. Though it places a weak restriction upon the rate with which the diameters of elements in the mesh may change, shape regularity allows for the locally refined meshes that are needed to resolve the singularities and other sharp local variations of the solution that occur in the majority of practical applications.

In the work NS74 of Nitsche and Schatz, local energy error estimates were established for interior subdomains under the assumption that the finite element grid is quasi-uniform. Such local energy estimates are helpful in understanding basic error behavior, especially "pollution effects" of global solution properties on local approximation quality, and they also provide an important technical tool in many proofs of pointwise bounds for the finite element method (cf. SW95]). In addition, the most relevant error notion in applications is often related to some local norm or functional instead of to the global energy error, as evidenced by the

Received by the editor August 14, 2008 and, in revised form, July 16, 2009.

2010 Mathematics Subject Classification. Primary 65N30, 65N15.

The first author was partially supported by NSF grant DMS-0713770.

The second author was partially supported by NSF grant DMS-0503050.

The third author was partially supported by NSF grant DMS-0612599. 
recent surge of interest in ensuring control of the error in calculating "quantities of interest" in adaptive finite element calculations instead of merely controlling the default global energy error (cf. [BR01]). As a final example of the applicability of local energy estimates, we mention that the estimates of [NS74 have been used to justify certain approaches to parallelization and adaptive meshing (cf. BH00] and XZ00]). Thus local energy estimates are of broad and fundamental importance in finite element theory.

Here we prove local energy error estimates under the assumption that the finite element triangulation is shape regular instead of under the more restrictive assumption of quasi-uniformity required in [NS74]. In other words, we essentially prove that the results of Nitsche and Schatz hold under the restrictions typically placed upon meshes in practical codes, which, in particular, allow for highly graded grids. Our main innovation is a novel "superapproximation" result which we state and prove in $\S 2$. In $\S 3$ we then prove a local energy bound that is valid on grids that are only assumed to be shape-regular. As in [NS74], our results are valid for operators that are only locally elliptic, so that the PDE under consideration may be degenerate or change type outside of the domain of interest. In contrast to [NS74, the results we present here allow for nonhomogeneous Neumann, Dirichlet, and mixed boundary conditions and also require only $L_{\infty}$ regularity of the coefficients of the differential operator. Finally, in $\S 4$ we compare our results with other local energy estimates appearing in the literature. These include, in particular, XZ00 and [NPV91, which contain local energy estimates on meshes satisfying conditions which allow for mesh grading but which exclude merely shape regular grids.

\section{AN IMPROVED SUPERAPPROXIMATION RESULT}

An essential feature of the proofs of local error estimates given in [NS74, and also of essentially all published proofs of local and maximum-norm a priori error estimates for finite element methods, is the use of superapproximation properties. In essence, superapproximation bounds establish that a function in the finite element space multiplied by any smooth function can be approximated exceptionally well by the finite element space.

In order to fix thoughts, we shall in this section assume for simplicity that $\Omega \subset \mathbb{R}^{n}$ is a polyhedral domain; a more general situation is considered in $\S 3$ below. Let $\mathcal{T}_{h}$ be a simplicial decomposition of $\Omega$. Denote by $h_{T}$ the diameter of the element $T \in \mathcal{T}_{h}$. We assume throughout that the elements in $\mathcal{T}_{h}$ are shape-regular, that is, each simplex $T \in \mathcal{T}_{h}$ contains a ball of diameter $c_{1} h_{T}$ and is contained in a ball of radius $C_{1} h_{T}$, where $c_{1}$ and $C_{1}$ are fixed. Also, let $S_{h}^{r}$ be a standard Lagrange finite element space consisting of continuous piecewise polynomials of degree $r-1$. We shall use standard notation for Sobolev spaces, norms, and seminorms, e.g., $\|u\|_{H^{1}(\Omega)}=\left(\int_{\Omega}\left(u^{2}+|\nabla u|^{2}\right) \mathrm{d} x\right)^{1 / 2},|u|_{W_{p}^{k}(\Omega)}=\left(\sum_{|\alpha|=k}\left\|D^{\alpha} u\right\|_{L_{p}(\Omega)}^{p}\right)^{1 / p}$, etc.

A standard superapproximation result is as follows. Let $\omega \in C^{\infty}(\Omega)$ with $|\omega|_{W_{\infty}^{j}(\Omega)} \leq C d^{-j}, 0 \leq j \leq r$. Then for each $\chi \in S_{h}^{r}$, there exists $\eta \in S_{h}^{r}$ such that for each $T \in \mathcal{T}_{h}$ satisfying $d \geq h_{T}$,

$$
\|\omega \chi-\eta\|_{H^{1}(T)} \leq C\left(\frac{h_{T}}{d}\|\nabla \chi\|_{L_{2}(T)}+\frac{h_{T}}{d^{2}}\|\chi\|_{L_{2}(T)}\right) .
$$

Our modified superapproximation estimate follows. This result, which is due to the authors jointly, has also appeared in a slightly different context in the work Guz06] of the second author. 
Theorem 2.1. Let $\omega \in C^{\infty}(\Omega)$ with $|\omega|_{W_{\infty}^{j}(\Omega)} \leq C d^{-j}$ for $0 \leq j \leq r$. Then for each $\chi \in S_{h}^{r}$, there exists $\eta \in S_{h}^{r}$ such that for each $T \in \mathcal{T}_{h}$ satisfying $d \geq h_{T}$,

$$
\left\|\omega^{2} \chi-\eta\right\|_{H^{1}(T)} \leq C\left(\frac{h_{T}}{d}\|\nabla(\omega \chi)\|_{L_{2}(T)}+\frac{h_{T}}{d^{2}}\|\chi\|_{L_{2}(T)}\right) .
$$

Remark 2.2. There are two differences between (2.1) and (2.2). First, in (2.1) we consider approximation of $\omega \chi$, whereas in (2.2) we consider approximation of $\omega^{2} \chi$. Second, in (2.1) the norms on the right-hand side involve only $\chi$, whereas in (2.2) the $H^{1}$ seminorm involves $\omega \chi$. If we think of $\omega$ as a cutoff function, this distinction becomes vitally important: $\omega \chi$ has the same support as $\omega^{2} \chi$, whereas the support of $\chi$ is generally larger than that of $\omega \chi$. This seemingly minor difference will allow us to establish local energy estimates on grids that are only assumed to be shape regular.

Proof. Let $I_{h}: C^{0}(\Omega) \rightarrow S_{h}^{r}$ be the standard Lagrange interpolant. We shall choose $\eta=I_{h}\left(\omega^{2} \chi\right)$ in (2.2). For $T \in \mathcal{T}_{h}$, we may use standard approximation theory (cf. BS02 ) to calculate

$$
\begin{aligned}
\left\|\omega^{2} \chi-I_{h}\left(\omega^{2} \chi\right)\right\|_{H^{1}(T)} & \leq C h_{T}^{n / 2}\left\|\omega^{2} \chi-I_{h}\left(\omega^{2} \chi\right)\right\|_{W_{\infty}^{1}(T)} \\
& \leq C h_{T}^{n / 2+r-1}\left|\omega^{2} \chi\right|_{W_{\infty}^{r}(T)} .
\end{aligned}
$$

Noting that $D^{\alpha} \chi=0$ for all multi-indices $\alpha$ with $|\alpha|=r$, recalling that $\frac{h_{T}}{d} \leq 1$, and employing inverse estimates, we compute

$$
\begin{aligned}
C h_{T}^{n / 2+r-1}\left|\omega^{2} \chi\right|_{W_{\infty}^{r}(T)} \leq C\left(\sum_{i=2}^{r} h_{T}^{i-1}\left|\omega^{2}\right|_{W_{\infty}^{i}(T)}\right)\|\chi\|_{L_{2}(T)} \\
+C h_{T}^{n / 2+r-1} \sum_{|\alpha|=1,|\beta|=r-1}\left\|D^{\alpha} \omega^{2} D^{\beta} \chi\right\|_{L_{\infty}(T)} \\
\leq C \frac{h_{T}}{d^{2}}\|\chi\|_{L_{2}(T)}+C h_{T}^{n / 2+r-1} \sum_{|\alpha|=1,|\beta|=r-1}\left\|D^{\alpha} \omega^{2} D^{\beta} \chi\right\|_{L_{\infty}(T)} .
\end{aligned}
$$

We next consider the terms $\left\|D^{\alpha} \omega^{2} D^{\beta} \chi\right\|_{L_{\infty}(T)}$ above. Since $|\alpha|=1$, we have $D^{\alpha} \omega^{2}=2 \omega D^{\alpha} \omega$. Let $\hat{\omega}=\frac{1}{|T|} \int_{T} \omega \mathrm{d} x$ so that $\|\omega-\hat{\omega}\|_{L_{\infty}(T)} \leq C h_{T}|\omega|_{W_{\infty}^{1}(T)} \leq$ $C \frac{h_{T}}{d}$. Employing inverse estimates, we thus have

$$
\begin{aligned}
C h_{T}^{n / 2+r-1} & \sum_{|\alpha|=1,|\beta|=r-1}\left\|D^{\alpha} \omega^{2} D^{\beta} \chi\right\|_{L_{\infty}(T)} \\
\leq & C d^{-1} h_{T}^{n / 2+r-1} \sum_{|\beta|=r-1}\left\|\omega D^{\beta} \chi\right\|_{L_{\infty}(T)} \\
\leq & C d^{-1} h_{T}^{n / 2+r-1} \sum_{|\beta|=r-1}\left(\left\|(\omega-\hat{\omega}) D^{\beta} \chi\right\|_{L_{\infty}(T)}+\left\|\hat{\omega} D^{\beta} \chi\right\|_{L_{\infty}(T)}\right) \\
\leq & C\left(\frac{h_{T}}{d^{2}}\|\chi\|_{L_{2}(T)}+\frac{h_{T}}{d}|\hat{\omega} \chi|_{H^{1}(T)}\right) \\
\leq & C\left(\frac{h_{T}}{d^{2}}\|\chi\|_{L_{2}(T)}+\frac{h_{T}}{d}|(\hat{\omega}-\omega) \chi|_{H^{1}(T)}+\frac{h_{T}}{d}|\omega \chi|_{H^{1}(T)}\right) .
\end{aligned}
$$


Using an inverse inequality, we find that

$$
\begin{aligned}
\frac{h_{T}}{d}|(\hat{\omega}-\omega) \chi|_{H^{1}(T)} & \leq \frac{h_{T}}{d}\left(|\omega|_{W_{\infty}^{1}(T)}\|\chi\|_{L_{2}(T)}+\|\hat{\omega}-\omega\|_{L_{\infty}(T)}|\chi|_{H^{1}(T)}\right) \\
& \leq C \frac{h_{T}}{d}\left(\frac{1}{d}\|\chi\|_{L_{2}(T)}+\frac{h_{T}}{d}|\chi|_{H^{1}(T)}\right) \\
& \leq C \frac{h_{T}}{d^{2}}\|\chi\|_{L_{2}(T)} .
\end{aligned}
$$

Inserting (2.6) into (2.5) and the result into (2.4) and (2.3) completes the proof of (2.2).

\section{Local $H^{1}$ estimates}

In this section we state and prove a local $H^{1}$ estimate that is valid on highly graded grids. We now let $\Omega$ be a domain in $\mathbb{R}^{n}$, and let $\Omega_{0}$ be a bounded subdomain of $\Omega$. We decompose $\partial \Omega \cap \partial \Omega_{0}$ (if it is nonempty) into a Dirichlet portion $\Gamma_{D}$ and a Neumann portion $\Gamma_{N}$. For the sake of simplicity, we assume that $\Gamma_{D}$ is polyhedral and that $\Gamma_{N}$ is either polyhedral or Lipschitz. Let $u$ satisfy

$$
\begin{aligned}
-\operatorname{div}(A \nabla u)+b \cdot \nabla u+c u & =f \text { in } \Omega_{0}, \\
u & =g_{D} \text { on } \Gamma_{D}, \\
\frac{\partial u}{\partial n_{A}} & =g_{N} \text { on } \Gamma_{N} .
\end{aligned}
$$

Here $A$ is an $n \times n$ coefficient matrix that is uniformly bounded and positive definite in $\Omega, b \in L_{\infty}\left(\Omega_{0}\right)^{n}, c \in L_{\infty}\left(\Omega_{0}\right)$, and $\frac{\partial}{\partial n_{A}}$ is the conormal derivative with respect to $A$. Note that we make no assumptions about the differential equation solved by $u$ outside of $\Omega_{0}$.

Let $H_{D, 0}^{1}\left(\Omega_{0}\right)=\left\{u \in H^{1}\left(\Omega_{0}\right):\left.u\right|_{\Gamma_{D}}=0\right\}$, and let $H_{D}^{1}\left(\Omega_{0}\right)=\left\{u \in H^{1}\left(\Omega_{0}\right)\right.$ : $\left.\left.u\right|_{\Gamma_{D}}=g_{D}\right\}$. Also, let $H_{<}^{1}(B)=\left\{u \in H^{1}\left(\Omega_{0}\right):\left.u\right|_{\Omega \backslash B}=0\right\}$ for subsets $B$ of $\Omega_{0}$. Thus functions in $H_{<}^{1}(B)$ are zero on $\partial B \backslash \partial \Omega$, but may be nonzero on portions of $\partial B$ coinciding with $\partial \Omega$, or put in other terms, functions in $H_{<}^{1}(B)$ are compactly supported in $B$ modulo $\partial \Omega$. Rewriting (3.1) in its weak form, we find that $u \in$ $H_{D}^{1}\left(\Omega_{0}\right)$ satisfies

$$
\begin{aligned}
L(u, v) & :=\int_{\Omega}(A \nabla u \nabla v+b \cdot \nabla u v+c u v) \mathrm{d} x \\
& =\int_{\Omega} f v \mathrm{~d} x+\int_{\Gamma_{N}} g_{N} v \mathrm{~d} \sigma, v \in H_{D, 0}^{1}\left(\Omega_{0}\right) \cap H_{<}^{1}\left(\Omega_{0}\right) .
\end{aligned}
$$

Following [NS74, we do not assume that $L$ is coercive over $H^{1}\left(\Omega_{0}\right)$, but rather we make a local coercivity assumption:

R1: Local coercivity. There exists a constant $d_{0}>0$ such that if $B$ is the intersection of any open sphere of diameter $d \leq d_{0}$ with $\Omega_{0}$, then $L$ is coercive over $H_{<}^{1}(B)$, that is, for some constant $C_{1}>0$,

$$
\left(C_{1}\right)^{-1}\|u\|_{H^{1}(B)}^{2} \leq L(u, u) \leq C_{1}\|u\|_{H^{1}(B)}^{2}, u \in H_{<}^{1}(B) .
$$

Remark 3.1. R1 may be satisfied in one of two ways. It may happen that $L$ is coercive over $H^{1}\left(\Omega_{0}\right)$, in which case no further argument is needed. R1 is also satisfied so long as a Poincaré inequality

$$
\|u\|_{L_{2}(B)} \leq C d\|u\|_{H^{1}(B)}, u \in H_{<}^{1}(B)
$$


holds for balls $B$ as in R1 having small enough diameter (cf. Remark 1.2 of [NS74]). Such Poincaré inequalities always hold for interior balls. If $B$ is the nontrivial intersection of an open ball with $\Omega$, then (3.4) holds for $d \leq d_{1}$ small enough under the restrictions we have placed on $\partial \Omega \cap \partial \Omega_{0}$; here $d_{1}$ depends on the properties of $\partial \Omega \cap \partial \Omega_{0}$.

Next, we make assumptions concerning the finite element approximation $u_{h}$ of $u$. Let $\mathcal{T}_{0}$ be the sub-triangulation of $\mathcal{T}_{h}$ such that $\Omega_{0} \subset \bigcup_{T \in \mathcal{T}_{0}} \bar{T}$ and $T \cap \Omega_{0} \neq \emptyset$ for all $T \in \mathcal{T}_{0}$. Let $h_{T}=\operatorname{diam}(T)$ for $T \in \mathcal{T}_{0}$. We denote our trial finite element space by $S_{D} . S_{D}$ may be thought of as a Lagrange finite space associated to the mesh $\mathcal{T}_{0}$, though following NS74 we will prove our results under abstract assumptions that potentially allow for other finite-dimensional spaces. We do not assume that $S_{D} \subset H_{D}^{1}\left(\Omega_{0}\right)$. In addition, we let $S_{D, 0}=S_{D} \cap H_{D, 0}^{1}\left(\Omega_{0}\right)$ be our test finite element space. We assume that $u_{h}$ is the local finite element approximation to $u$ on $\Omega_{0}$, that is, $u_{h} \in S_{D}$ and

$$
L\left(u-u_{h}, v_{h}\right)=0 \text { for all } v_{h} \in S_{D, 0} \cap H_{<}^{1}\left(\Omega_{0}\right) .
$$

We do not explicitly fix $u_{h}$ on the Dirichlet portion of the boundary, but rather implicitly assume that $\left.u_{h}\right|_{\Gamma_{D}}$ is set equal to some appropriate interpolant or projection of $g_{D}$.

Next, we state properties that $S_{D}$ and $S_{D, 0}$ must possess in order to prove the desired local energy error estimate. Let $\tilde{d} \leq d_{0}$ be a fixed parameter, and let $G_{1}$ and $G$ be arbitrary subsets of $\Omega_{0}$ with $G_{1} \subset G$ and $\operatorname{dist}\left(G_{1}, \partial G \backslash \partial \Omega\right)=\tilde{d}>0$. Then the following are assumed to hold:

A1: Local interpolant. There exists a local interpolant $I$ such that for each $u \in H_{<}^{1}\left(G_{1}\right) \cap C\left(G_{1}\right), I u \in S_{D} \cap H_{<}^{1}(G)$, and for each $u \in H_{D, 0}^{1}\left(\Omega_{0}\right), I u \in S_{D, 0}$.

A2: Inverse properties. For each $\chi \in S_{D}, T \in \mathcal{T}_{0}, 1 \leq p \leq q \leq \infty$, and $0 \leq \nu \leq s \leq r$ with $r$ sufficiently small,

$$
\|\chi\|_{W_{q}^{s}(T)} \leq C h_{T}^{\nu-s+\frac{n}{p}-\frac{n}{q}}\|\chi\|_{W_{p}^{\nu}(T)} .
$$

A3: Superapproximation. Let $\omega \in C^{\infty}\left(\Omega_{0}\right) \cap H_{<}^{1}\left(G_{1}\right)$ with $|\omega|_{W_{\infty}^{j}\left(\Omega_{0}\right)} \leq C d^{-j}$ for integers $0 \leq j \leq r$ with $r$ sufficiently large. For each $\chi \in S_{D, 0}$ and for each $T \in \mathcal{T}_{0}$ satisfying $h_{T} \leq d$,

$$
\left\|\omega^{2} \chi-I\left(\omega^{2} \chi\right)\right\|_{H^{1}(T)} \leq C\left(\frac{h_{T}}{d}\|\nabla(\omega \chi)\|_{L_{2}(T)}+\frac{h_{T}}{d^{2}}\|\chi\|_{L_{2}(T)}\right),
$$

where the interpolant $I$ is as in A1 above.

Remark 3.2. A1, A2, and A3 are satisfied by standard Lagrange finite element spaces defined on shape-regular triangular grids. A1 also essentially requires that the finite element mesh resolve $G \backslash G_{1}$, i.e., that $\tilde{d} \geq K \max _{T \cap G \neq \emptyset} h_{T}$ with $K$ large enough.

We begin by proving a Caccioppoli-type estimate for "discrete harmonic" functions. Such a statement was also proved in NS74 as a preliminary to local energy estimates, though the proof we give below more closely follows [SW77.

Lemma 3.3. Let $G_{0} \subset G \subset \Omega_{0}$ be given, and let $\operatorname{dist}\left(G_{0}, \partial G \backslash \partial \Omega\right)=d$ with $d \leq \frac{d_{0}}{2}$ where $d_{0}$ is the parameter defined in the assumption R1. Also, let A1, A2, and A3 hold with $\tilde{d}=\frac{d}{4}$, and assume that $u_{h} \in S_{D, 0}$ satisfies

$$
L\left(u_{h}, v_{h}\right)=0 \text { for all } v_{h} \in S_{D, 0} \cap H_{<}^{1}(G) .
$$


In addition, let $\max _{T \cap G \neq \emptyset} \frac{h_{T}}{d} \leq \frac{1}{4}$. Then

$$
\left\|u_{h}\right\|_{H^{1}\left(G_{0}\right)} \leq C \frac{1}{d}\left\|u_{h}\right\|_{L_{2}(G)} .
$$

Here $C$ depends only on the constants in (3.6) and (3.7) and the coefficients of $L$.

Proof. We assume that $G_{0}$ is the intersection of a ball $B_{\frac{d}{4}}$ of radius $\frac{d}{4}$ with $\Omega_{0}$; the general case may be proved using a covering argument. Let then $G_{1}$ and $G_{2}$ be the intersections with $\Omega_{0}$ of balls having the same center as $G_{0}$ and having radii $\frac{d}{2}$ and $\frac{3 d}{4}$, respectively, and without loss of generality let $G$ be the intersection of the corresponding ball of radius $d$ with $\Omega_{0}$. Then let $\omega \in C^{\infty}\left(G_{1}\right) \cap H_{<}^{1}\left(G_{1}\right)$ be a cutoff function which is 1 on $G_{0}$ and which satisfies $\|\omega\|_{W_{\infty}^{j}\left(G_{1}\right)} \leq C d^{-j}, 0 \leq j \leq r$. We may then apply the assumptions A1 through A3 to the pairs $G_{1}$ and $G_{2}$, and $G_{2}$ and $G$.

Using (3.3), we first compute that

$$
\left\|u_{h}\right\|_{H^{1}\left(G_{0}\right)}^{2} \leq\left\|\omega u_{h}\right\|_{H^{1}(G)}^{2} \leq C L\left(\omega u_{h}, \omega u_{h}\right) .
$$

Using the fact that $\|\nabla \omega\|_{L_{\infty}(\Omega)} \leq \frac{C}{d}$, we compute that for any $\epsilon>0$,

$$
\begin{aligned}
& L\left(\omega u_{h}, \omega u_{h}\right)=L\left(u_{h}, \omega^{2} u_{h}\right) \\
& \quad+\int_{\Omega} u_{h}\left[-A \nabla\left(\omega u_{h}\right) \nabla \omega+u_{h} A \nabla \omega \nabla \omega+A \nabla \omega \nabla\left(\omega u_{h}\right)+\omega u_{h} b \cdot \nabla \omega\right] \mathrm{d} x \\
& \quad \leq\left|L\left(u_{h}, \omega^{2} u_{h}\right)\right|+C \frac{1}{d^{2} \epsilon}\left\|u_{h}\right\|_{L_{2}(G)}^{2}+\epsilon\left\|\omega u_{h}\right\|_{H_{1}(G)}^{2} .
\end{aligned}
$$

Next we use (3.8) and (3.7) to compute

$$
\begin{aligned}
L\left(u_{h}, \omega^{2} u_{h}\right) & =L\left(u_{h}, \omega^{2} u_{h}-I\left(\omega^{2} u_{h}\right)\right) \\
& \leq C \sum_{T \cap G_{2} \neq \emptyset} h_{T}\left\|u_{h}\right\|_{H^{1}(T)}\left(\frac{1}{d}\left|\omega u_{h}\right|_{H^{1}(T)}+\frac{1}{d^{2}}\left\|u_{h}\right\|_{L_{2}(T)}\right) .
\end{aligned}
$$

Using (3.6) and the fact that $\frac{h_{T}}{d} \leq 1$, we have for $\epsilon$ as above that

$$
\begin{gathered}
C h_{T}\left\|u_{h}\right\|_{H^{1}(T)}\left(\frac{1}{d}\left|\omega u_{h}\right|_{H^{1}(T)}+\frac{1}{d^{2}}\left\|u_{h}\right\|_{L_{2}(T)}\right) \\
\leq \frac{C}{\epsilon d^{2}}\left\|u_{h}\right\|_{L_{2}(T)}^{2}+\epsilon\left|\omega u_{h}\right|_{H^{1}(T)}^{2} .
\end{gathered}
$$

Inserting (3.13) into (3.12), noting that $T \cap G_{2} \neq \emptyset$ implies that $T \subset G$ (since $\max _{T \cap G \neq \emptyset} h_{T} \leq \frac{d}{4}$ ) yields that for $\epsilon>0$,

$$
L\left(u_{h}, \omega^{2} u_{h}\right) \leq \frac{C}{\epsilon d^{2}}\left\|u_{h}\right\|_{L_{2}(G)}^{2}+\epsilon\left\|\omega u_{h}\right\|_{H^{1}(G)}^{2} .
$$

Inserting (3.14) into (3.11) and the result into (3.10) yields

$$
\left\|\omega u_{h}\right\|_{H^{1}(G)}^{2} \leq \frac{C}{\epsilon d^{2}}\left\|u_{h}\right\|_{L_{2}(G)}^{2}+2 \epsilon\left\|\omega u_{h}\right\|_{H^{1}(G)}^{2} .
$$

Taking $\epsilon=\frac{1}{4}$ so that we may kick back the last term above and inserting the result into (3.10) then completes the proof of (3.9).

We now prove a local energy error estimate. In our proof below we shall follow NS74 by using a local finite element projection in order to split the finite element error into an approximation error and a "discrete harmonic" term which may be bounded using Lemma 3.3. We note, however, that the use of a local finite element 
projection is not necessary, and our final local error estimate may in fact be proved with some simple modifications to the proof of Lemma 3.3 above. These two styles of proof are essentially equivalent. Local finite element projections have been used, for example, in [NS74], SW77], SW95, and [AL95] in order to prove local a priori error estimates. The methodology of Lemma 3.3 in which no local projections are used has been employed, for example, in Dem04 and Guz06] in order to prove local a priori error estimates and in [XZ00], [LN03], and [Dem07] in order to prove local a posteriori error estimates.

Theorem 3.4. Let $G_{0} \subset G \subset \Omega_{0}$ be given, and let $\operatorname{dist}\left(G_{0}, \partial G \backslash \partial \Omega\right)=d$ with $d \leq \min \left\{\frac{d_{0}}{2}, d_{1}\right\}$ where $d_{0}$ is the parameter defined in the assumption $R 1$ and $d_{1}$ is defined in Remark 3.1. Also, let A1, A2, and A3 hold with $\tilde{d}=\frac{d}{16}$. In addition, let $\max _{T \cap G \neq \emptyset} \frac{h_{T}}{d} \leq \frac{1}{16}$. Then

$$
\begin{aligned}
\left\|u-u_{h}\right\|_{H^{1}\left(G_{0}\right) \leq} & C \min _{u_{h}-\chi \in S_{D, 0}}\left(\|u-\chi\|_{H^{1}(G)}+\frac{1}{d}\|u-\chi\|_{L_{2}(G)}\right) \\
& +C \frac{1}{d}\left\|u-u_{h}\right\|_{L_{2}(G)} .
\end{aligned}
$$

Here $C$ depends only on the constant $C$ in (2.2) and the coefficients of $L$.

Proof. We assume that $G_{0}$ is the intersection of a ball $B_{\frac{d}{2}}$ of radius $\frac{d}{2}$ with $\Omega_{0}$; the general case may be proved using a covering argument. Let $G_{1}$ be the intersection with $\Omega_{0}$ of a ball having the same center as $G_{0}$ and having radius $\frac{3 d}{4}$, and without loss of generality let $G$ be the intersection of the corresponding ball of radius $d$ with $\Omega_{0}$. Then let $\omega \in C^{\infty}(G) \cap H_{<}^{1}(G)$ be a cutoff function which is 1 on $G_{1}$ and which satisfies $\|\omega\|_{W_{\infty}^{j}(G)} \leq C d^{-j}, 0 \leq j \leq r$. Note that since $\operatorname{dist}\left(G_{0}, \partial G_{1} \backslash \partial \Omega\right)=\frac{d}{4}$, the assumptions that $\max _{T \cap G \neq \emptyset} \frac{h_{T}}{d} \leq \frac{1}{16}$ and A1, A2, and A3 hold with $\tilde{d}=\frac{d}{16}$ allow us to apply Lemma 3.3 with $G_{0}$ on the left-hand side of the estimate (3.9) and $G_{1}$ on the right-hand side.

We now proceed assuming momentarily that $u_{h} \in S_{D, 0}$. We let $P(\omega u)$ be a local finite element projection of $\omega u$. In particular, let $P(\omega u) \in S_{D, 0} \cap H_{<}^{1}(G)$ satisfy

$$
L\left(\omega u-P(\omega u), v_{h}\right)=0, v_{h} \in S_{D, 0} \cap H_{<}^{1}(G) .
$$

Since $\operatorname{diam}(G) \leq 2 d \leq d_{0}$, we may apply the local coercivity condition (3.3). The relation (3.17) and (3.3) then imply the stability estimate

$$
\|P(\omega u)\|_{H^{1}(G)} \leq C\|\omega u\|_{H^{1}(G)}
$$

Employing (3.9) and using (3.4) while recalling that $\omega \equiv 1$ on $G_{1}$, we compute that

$$
\begin{aligned}
\| u- & u_{h}\left\|_{H^{1}\left(G_{0}\right)} \leq\right\| \omega u-P(\omega u)\left\|_{H^{1}\left(G_{0}\right)}+\right\| P(\omega u)-u_{h} \|_{H^{1}\left(G_{0}\right)} \\
& \leq\|\omega u-P(\omega u)\|_{H^{1}(G)}+\frac{C}{d}\left\|P(\omega u)-u_{h}\right\|_{L_{2}\left(G_{1}\right)} \\
& \leq\|\omega u-P(\omega u)\|_{H^{1}(G)}+\frac{C}{d}\left(\|P(\omega u)-\omega u\|_{L_{2}\left(G_{1}\right)}+\left\|u-u_{h}\right\|_{L_{2}\left(G_{1}\right)}\right) \\
& \leq C\|\omega u-P(\omega u)\|_{H^{1}(G)}+\frac{C}{d}\left\|u-u_{h}\right\|_{L_{2}\left(G_{1}\right)} .
\end{aligned}
$$


Next, we use the triangle inequality and (3.18) while recalling that $\|\omega\|_{W_{\infty}^{j}\left(G_{2}\right)} \leq$ $C d^{-j}$ in order to find that

$$
\begin{aligned}
\|\omega u-P(\omega u)\|_{H^{1}(G)} & \leq C\|\omega u\|_{H^{1}(G)} \\
& \leq C\left(\|u\|_{H^{1}(G)}+\frac{1}{d}\|u\|_{L_{2}(G)}\right) .
\end{aligned}
$$

Inserting (3.20) into (3.19) yields that for $u_{h} \in S_{D, 0} \cap H_{<}^{1}(G)$,

$$
\left\|u-u_{h}\right\|_{H^{1}\left(G_{0}\right)} \leq C\left(\|u\|_{H^{1}(G)}+\frac{1}{d}\|u\|_{L_{2}(G)}+\frac{1}{d}\left\|u-u_{h}\right\|_{L_{2}(G)}\right) .
$$

In order to complete the proof of (3.16), we now drop the restriction that $u_{h} \in$ $S_{D, 0}$ and assume merely that $u_{h} \in S_{D}$. Let $\chi \in S_{D}$ with $u_{h}-\chi \in S_{D, 0}$ be as in the right-hand side of (3.16). We may then write $u-u_{h}=(u-\chi)-\left(u_{h}-\chi\right)$ and employ (3.21) with $u$ taken to be $u-\chi$ and $u_{h}$ taken to be $u_{h}-\chi$, since $u_{h}-\chi \in S_{D, 0}$. Doing so yields precisely (3.16), which completes the proof of the theorem.

\section{Remarks on Literature}

We conclude our presentation with some remarks concerning previous proofs of local energy estimates that have appeared in the literature. In the proof given in [NS74, the standard superapproximation estimate (2.1) is in essence inserted into (3.12) and elementary manipulations are then used to obtain

$$
\begin{aligned}
L\left(u_{h}, \omega^{2} u_{h}\right) & \leq C \sum_{T \cap G_{2} \neq \emptyset} \frac{h_{T}}{d}\left\|u_{h}\right\|_{H_{1}(T)}^{2}+\frac{1}{d^{2}}\left\|u_{h}\right\|_{L_{2}(T)} \\
& \leq \frac{h}{d}\left\|u_{h}\right\|_{H^{1}(G)}^{2}+\frac{1}{d^{2}}\left\|u_{h}\right\|_{L_{2}(G)}^{2},
\end{aligned}
$$

where $h=\max _{T \cap G \neq \emptyset} h_{T}$. The resulting estimate for discrete harmonic functions $u_{h}$ is then

$$
\left\|u_{h}\right\|_{H^{1}\left(G_{0}\right)}^{2} \leq C \frac{h}{d}\left\|u_{h}\right\|_{H^{1}(G)}^{2}+\frac{1}{d^{2}}\left\|u_{h}\right\|_{L_{2}(G)} .
$$

In [NS74, (4.2) is iterated once in order to obtain enough powers of $h$ to apply an inverse inequality to the term $\left\|u_{h}\right\|_{H^{1}(G)}^{2}$. In NPV91] and [XZ00] the condition $\underline{h} \geq C h^{\gamma}$ is imposed, where $\underline{h}=\min _{T \in \mathcal{T}_{h}} h_{T}$ and $\gamma \geq 1(\gamma=1$ yields a quasiuniform grid). (4.2) can then be iterated enough times (with the necessary number of iterations depending on $\gamma$ ) in order to similarly apply an inverse inequality. If $\gamma>1$, meshes with nontrivial grading are allowed, but merely shape regular grids are still not admitted. In addition, the constants in the resulting estimate depend on $\gamma$ (the amount of mesh grading) as well as shape regularity properties. With use of the improved superapproximation estimate given in $\S 2$, we completely avoid iterating inequalities, inverse estimates need only be applied elementwise, and the constants in the resulting estimate depend only on shape regularity properties and not on the amount of mesh grading.

In STW90, the third author gave a proof of local estimates for quasi-uniform grids, and for piecewise linear elements only, which in some ways anticipates the proof we give here. When $\chi$ is piecewise linear, the improved superapproximation result (2.2) is much easier to obtain. Though this observation is not stated explicitly, it is in a sense used implicitly in STW90 in order to avoid the iteration step mentioned above in the case of piecewise linear elements. However, the use of 
improved superapproximation is not rigorously justified in STW90 even for linear elements, and the article also does not make the connection between elimination of the iteration step and extension of local estimates to shape regular grids.

\section{ACKNOWLEDGMENTS}

The authors would like to acknowledge an anonymous referee whose comments led to an improved presentation.

\section{REFERENCES}

[AL95] Douglas N. Arnold and Xiao Bo Liu, Local error estimates for finite element discretizations of the Stokes equations, RAIRO Modél. Math. Anal. Numér. 29 (1995), no. 3, 367-389. MR.1342712 (96d:76055)

[BH00] Randolph E. Bank and Michael Holst, A new paradigm for parallel adaptive meshing algorithms, SIAM J. Sci. Comput. 22 (2000), no. 4, 1411-1443 (electronic). MR 1797889 (2002g:65117)

[BR01] Roland Becker and Rolf Rannacher, An optimal control approach to a posteriori error estimation in finite element methods, Acta Numer. 10 (2001), 1-102. MR2 009692

[BS02] Susanne C. Brenner and L. Ridgway Scott, The mathematical theory of finite element methods, second ed., Texts in Applied Mathematics, vol. 15, Springer-Verlag, New York, 2002. MR.1894376 (2003a:65103)

[Dem04] Alan Demlow, Localized pointwise error estimates for mixed finite element methods, Math. Comp. 73 (2004), no. 248, 1623-1653 (electronic). MR2059729 (2005e:65184)

[Dem07] Local a posteriori estimates for pointwise gradient errors in finite element methods for elliptic problems, Math. Comp. 76 (2007), no. 257, 19-42 (electronic). MR.2261010

[Guz06] Johnny Guzmán, Pointwise error estimates for discontinuous Galerkin methods with lifting operators for elliptic problems, Math. Comp. 75 (2006), no. 255, 1067-1085 (electronic). MR 2219019 (2006m:65269)

[LN03] Xiaohai Liao and Ricardo H. Nochetto, Local a posteriori error estimates and adaptive control of pollution effects, Numer. Methods Partial Differential Equations 19 (2003), no. 4, 421-442. MR.1980188(2004c:65130)

[NPV91] R. H. Nochetto, M. Paolini, and C. Verdi, An adaptive finite element method for twophase Stefan problems in two space dimensions. I. Stability and error estimates, Math. Comp. 57 (1991), no. 195, 73-108, S1-S11. MR.1079028 (92a:65322)

[NS74] Joachim A. Nitsche and Alfred H. Schatz, Interior estimates for Ritz-Galerkin methods, Math. Comp. 28 (1974), 937-958. MR51:9525

[STW90] Alfred H. Schatz, Vidar Thomée, and Wolfgang L. Wendland, Mathematical theory of finite and boundary element methods, DMV Seminar, vol. 15, Birkhäuser Verlag, Basel, 1990. MR:1116555 (92f:65004)

[SW77] Alfred H. Schatz and Lars B. Wahlbin, Interior maximum norm estimates for finite element methods, Math. Comp. 31 (1977), no. 138, 414-442. MR0431753 (55:4748)

[SW95] - Interior maximum-norm estimates for finite element methods, Part II, Math. Comp. 64 (1995), no. 211, 907-928. MR1297478 (95j:65143)

[XZ00] Jinchao Xu and Aihui Zhou, Local and parallel finite element algorithms based on two-grid discretizations, Math. Comp. 69 (2000), no. 231, 881-909. MR.1654026 (2000j:65102)

Department of Mathematics, University of Kentucky, 715 Patterson Office Tower, Lexington, Kentucky 40506-0027

E-mail address: demlow@ms.uky.edu

Division of Applied Mathematics, Brown University, 182 George Street, Providence, RHODE ISLAND 02906

E-mail address: johnny_guzman@brown.edu

Department of Mathematics, Malott Hall, Cornell University, Ithaca, New York 14853 